\title{
Leptonic and semileptonic B decays with tau at the B factories
}

\section{Yasuyuki Horii}

Kobayashi-Maskawa Institute, Nagoya University

E-mail: yhorii@hepl.phys.nagoya-u.ac.jp

\begin{abstract}
The leptonic decay $B^{-} \rightarrow \tau^{-} \overline{\mathrm{v}}_{\tau}$ and the semileptonic decays $B \rightarrow D^{(*)} \tau^{-} \overline{\mathrm{v}}_{\tau}$ provide opportunities for testing the Standard Model and for searching for new physics. Extended models including a charged Higgs boson are sensitive to these decays. In this report, recent results obtained at the B factories are introduced. Several results show the discrepancies from the expectations from the Standard Model.
\end{abstract}

The XIth International Conference on Heavy Quarks and Leptons,

June 11-15, 2012

Prague, Czech Republic 


\section{Introduction}

The leptonic decay $B^{-} \rightarrow \tau^{-} \overline{\mathrm{v}}_{\tau}$ is mediated by the $W^{-}$boson in the Standard Model (SM). ${ }^{1}$ The branching fraction $\mathscr{B}\left(B^{-} \rightarrow \tau^{-} \bar{v}_{\tau}\right)$ is a good probe for the parameters $f_{B}$ and $\left|V_{u b}\right|$, where $f_{B}$ is the $B^{-}$meson decay constant and $\left|V_{u b}\right|$ is the magnitude of a Cabibbo-Kobayashi-Maskawa (CKM) matrix element [1, 2]. The decay $B^{-} \rightarrow \tau^{-} \bar{v}_{\tau}$ could also be mediated by charged particles such as a charged Higgs boson in extended models [3, 4]. Because of the larger mass of the $\tau^{-}$lepton compared to the other leptons, this decay is relatively sensitive to various models with extended Higgs sector.

The semileptonic decays $B \rightarrow D^{(*)} \tau^{-} \bar{v}_{\tau}$, where the $B^{-}$and $\bar{B}^{0}$ decays are implied, are also mediated by the $W^{-}$boson in the SM. These decays are related to the form factors, which parametrize the hadronic interactions depending on $q^{2}=\left(p_{B}-p_{D^{(*)}}\right)^{2}$, and the magnitude of a CKM matrix element $\left|V_{c b}\right|$. These decays could also be affected by charged particles such as a charged Higgs boson in extended models [5, 6, 7, 8, 9]. Additional observables sensitive to new physics, such as the $\tau^{-}$polarization, are possibly provided from these decays.

Various measurements on the decays $B^{-} \rightarrow \tau^{-} \bar{v}_{\tau}$ and $B \rightarrow D^{(*)} \tau^{-} \bar{v}_{\tau}$ have been performed at the B factories. In this report, we introduce recent results obtained at the Belle [10] and BaBar [11] experiments.

\footnotetext{
${ }^{1}$ The charge-conjugate decays are implied throughout this report unless otherwise stated.
} 


\section{Recent results on $B^{-} \rightarrow \tau^{-} \bar{v}_{\tau}$}

The decay $B^{-} \rightarrow \tau^{-} \bar{v}_{\tau}$ includes multiple neutrinos in the final state considering the following $\tau^{-}$decays. At the $\mathrm{B}$ factories, it is exploited that a $B$ meson pair is generated from the process $e^{+} e^{-} \rightarrow \Upsilon(4 S) \rightarrow B \bar{B}$. We reconstruct one of the $B$ mesons (referred to as $B_{\text {tag }}$ ) in the event using the decays which have relatively larger decay rate with smaller background rate, and we compare the properties of the remaining particles (referred to as $B_{\text {sig }}$ ) to those expected for signal and background.

Two independent methods for reconstructing the $B_{\text {tag }}$ candidates are used for the analysis of the decay $B^{-} \rightarrow \tau^{-} \bar{v}_{\tau}$. One is the "hadronic" tag to fully reconstruct the $B_{\text {tag }}$ candidates from hadronic decays such as $B^{-} \rightarrow D^{0} \pi^{-}$, while the other is the "semileptonic" tag to reconstruct the $B_{\text {tag }}$ candidates from semileptonic decays $B^{-} \rightarrow D^{(*)} \ell^{-} \bar{v}_{\ell}(\ell=\mu$ or $e$ ). The efficiency for reconstructing the $B_{\text {tag }}$ candidates is higher for the semileptonic tag, while the purity is higher for the hadronic tag. Resulting sensitivity is comparable between the hadronic and semileptonic tags.

In the events where $B_{\text {tag }}$ candidates are reconstructed, we search for the $B^{-} \rightarrow \tau^{-} \bar{v}_{\tau}$ decays. The $\tau^{-}$lepton is identified in the decay channels $e^{-} \bar{v}_{e} \nu_{\tau}, \mu^{-} \bar{v}_{\mu} \nu_{\tau}, \pi^{-} \nu_{\tau}$, and so on, which cover most of the $\tau^{-}$decay rate [12]. After reconstructing the particles from the $\tau^{-}$decays except for the neutrinos, it is required that there are neither extra charged tracks nor extra $\pi^{0}$ candidates. After applying requirements on the kinematic variables for suppressing the backgrounds, signal yields are extracted using the extra energy detected in the electromagnetic calorimeter. For signal events, the extra energy must be either zero or a small value arising from beam background and from splitoff showers from $B_{\text {tag }}$ and $B_{\text {sig. }}$. On the other hand, background events are distributed toward higher value due to the contribution from additional neutral clusters.

The first evidence of the decay $B^{-} \rightarrow \tau^{-} \bar{v}_{\tau}$ was obtained by the Belle collaboration using hadronic tag for the $449 \times 10^{6} B \bar{B}$ data sample [13]. The measured branching fraction is $\mathscr{B}\left(B^{-} \rightarrow \tau^{-} \bar{v}_{\tau}\right)=\left[1.79_{-0.49}^{+0.56}(\text { stat })_{-0.51}^{+0.46}(\right.$ syst $\left.)\right] \times 10^{-4}$, where the significance is 3.5 standard deviations $(\sigma)$. Using semileptonic tag for the $657 \times 10^{6} B \bar{B}$ data sample, the Belle collaboration confirmed their result and obtain $\mathscr{B}\left(B^{-} \rightarrow \tau^{-} \bar{v}_{\tau}\right)=\left[1.54_{-0.37}^{+0.38}(\text { stat })_{-0.31}^{+0.29}(\right.$ syst $\left.)\right] \times 10^{-4}$ with a significance of $3.6 \sigma$ [14]. The BaBar collaboration also reported the results using hadronic tag for the $468 \times 10^{6} B \bar{B}$ data sample [15] and using semileptonic tag for the $459 \times 10^{6} B \bar{B}$ data sample [16] to evaluate $\mathscr{B}\left(B^{-} \rightarrow \tau^{-} \bar{v}_{\tau}\right)=\left[1.80_{-0.54}^{+0.57}(\right.$ stat $) \pm 0.26($ syst $\left.)\right] \times 10^{-4}$ and $\mathscr{B}\left(B^{-} \rightarrow \tau^{-} \bar{v}_{\tau}\right)=$ $[1.7 \pm 0.8$ (stat) \pm 0.2 (syst) $] \times 10^{-4}$, respectively. The former corresponds to a significance of $3.3 \sigma$. Figures 1 and 2 show the distributions of the extra energy obtained by the Belle and BaBar colaborations, respectively.

Figure 3 summarizes the results on the decay $B^{-} \rightarrow \tau^{-} \bar{v}_{\tau}$. A world average is calculated by the HFAG group to be $\mathscr{B}\left(B^{-} \rightarrow \tau^{-} \bar{v}_{\tau}\right)=(1.67 \pm 0.30) \times 10^{-4}$ [17]. The obtained value is consistent within $2 \sigma$ with a SM expectation $\mathscr{B}\left(B^{-} \rightarrow \tau^{-} \bar{v}_{\tau}\right)=(0.96 \pm 0.25) \times 10^{-4}$ based on $f_{B}$ obtained from lattice calculation [18] and $\left|V_{u b}\right|$ obtained from $b \rightarrow u$ transitions [12]. On the other hand, as shown in Figure 4 there is a discrepancy with a level of more than $2.5 \sigma$ from an expectation $\mathscr{B}\left(B^{-} \rightarrow \tau^{-} \bar{v}_{\tau}\right)=\left(0.73_{-0.07}^{+0.12}\right) \times 10^{-4}$ based on $\left|V_{u b}\right|$ obtained from a global fit on the CKM matrix elements assuming the SM [19]. To establish the signal and test the consistency with the SM and the extended models, we need further studies with more statistics and better analysis techniques. 

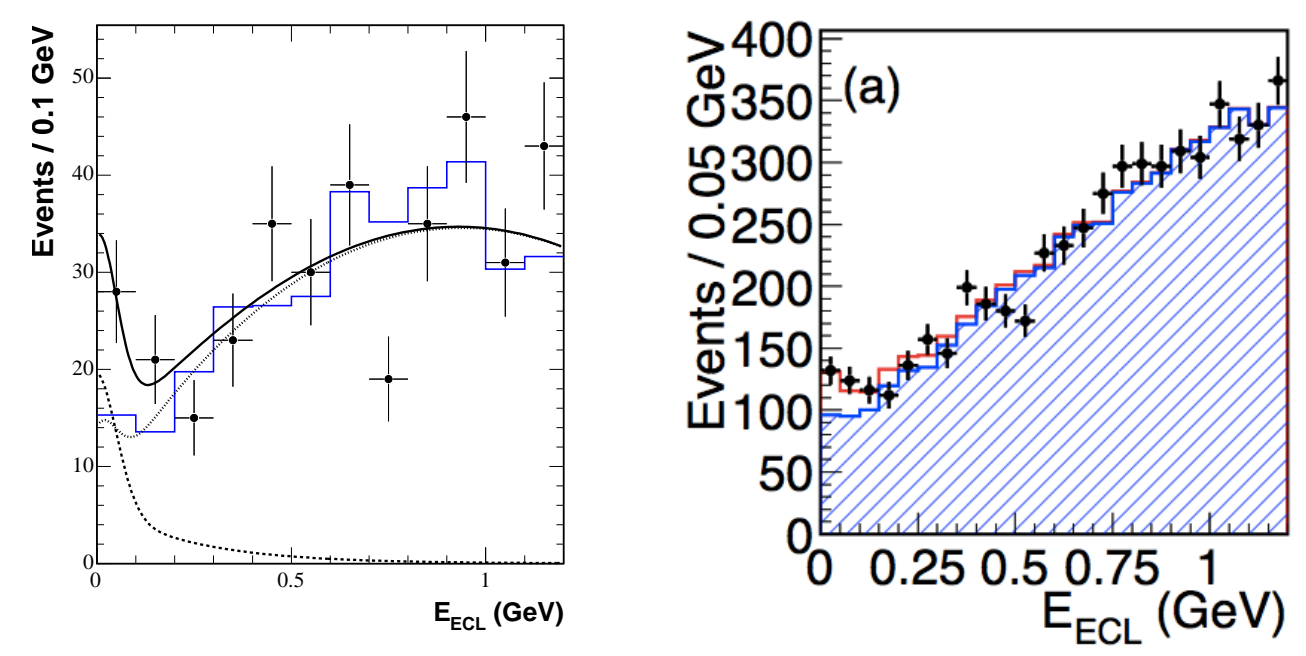

Figure 1: Signal extraction for the decay $B^{-} \rightarrow \tau^{-} \bar{v}_{\tau}$ based on the hadronic tag (left) and the semileptonic tag (right) at the Belle experiment. For the left figure, the data and the background MC samples are represented by the dots with error bars and the blue solid histogram, respectively. The solid curve shows the fit result with the sum of the signal (dashed curve) and the background (dotted curve) contributions. For the right figure, the data is represented by the dots with error bars. The blue hatched and the red solid histograms show the fit result for the background and signal contributions, respectively.
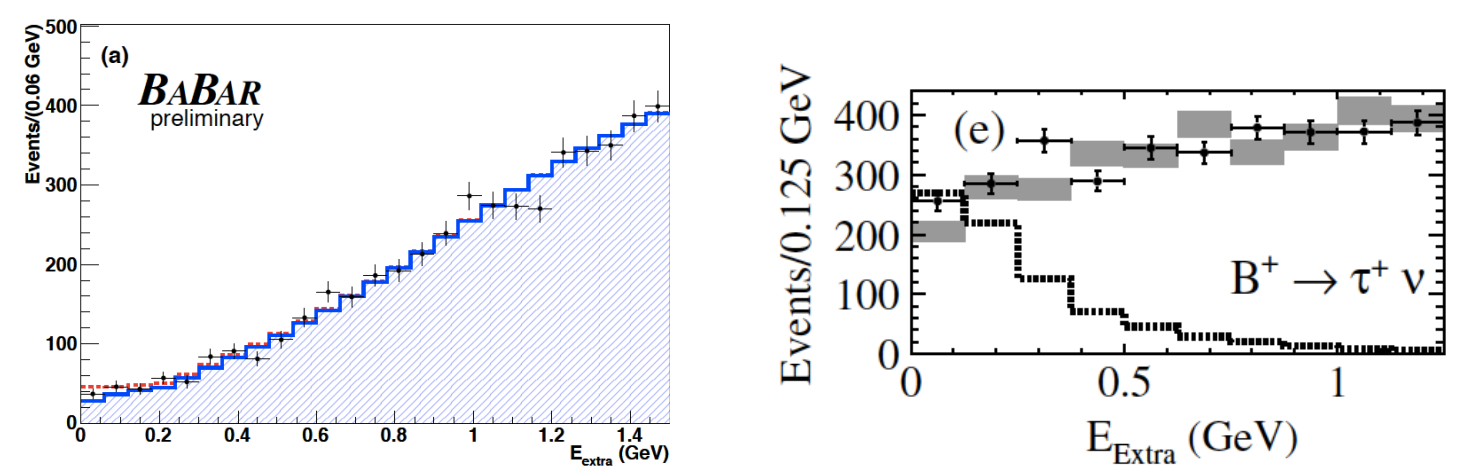

Figure 2: Signal extraction for the decay $B^{-} \rightarrow \tau^{-} \bar{v}_{\tau}$ based on the hadronic tag (left) and the semileptonic tag (right) at the BaBar experiment. The dots with error bars are the data for both figures. For the left figure, the blue hatched and the red dashed histograms are the background and the signal components, respectively, of the fit result. For the right figure, the gray shaded and the dotted histograms indicate the background MC simulation and the signal MC simulation normalized to 10 times the expected branching fraction. For the semileptonic tag, the signal yield is obtained from counted number of events by subtracting the background yield. 
HFAG average: $\mathcal{B}=(1.67 \pm 0.30) \times 10^{-4}$

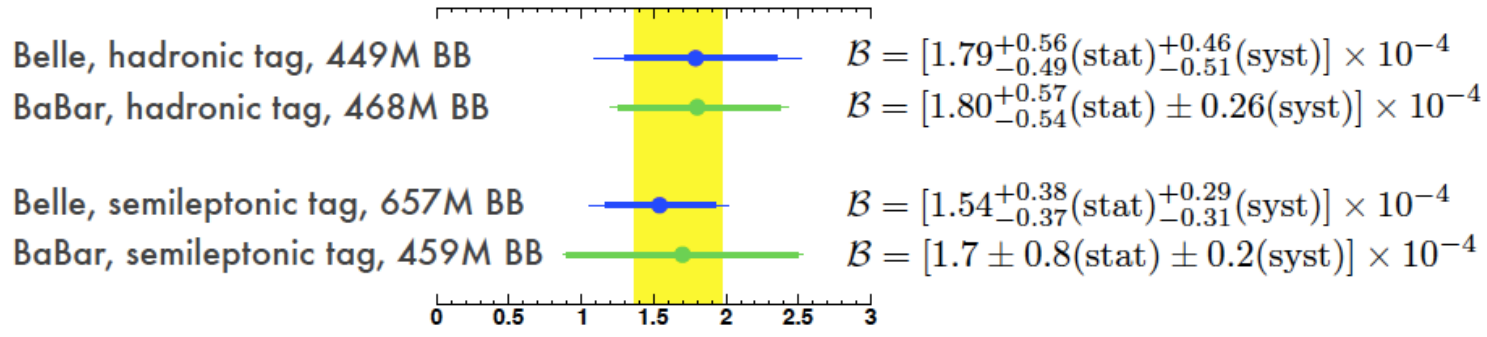

Figure 3: Summary of the results on the decay $B^{-} \rightarrow \tau^{-} \bar{v}_{\tau}$.

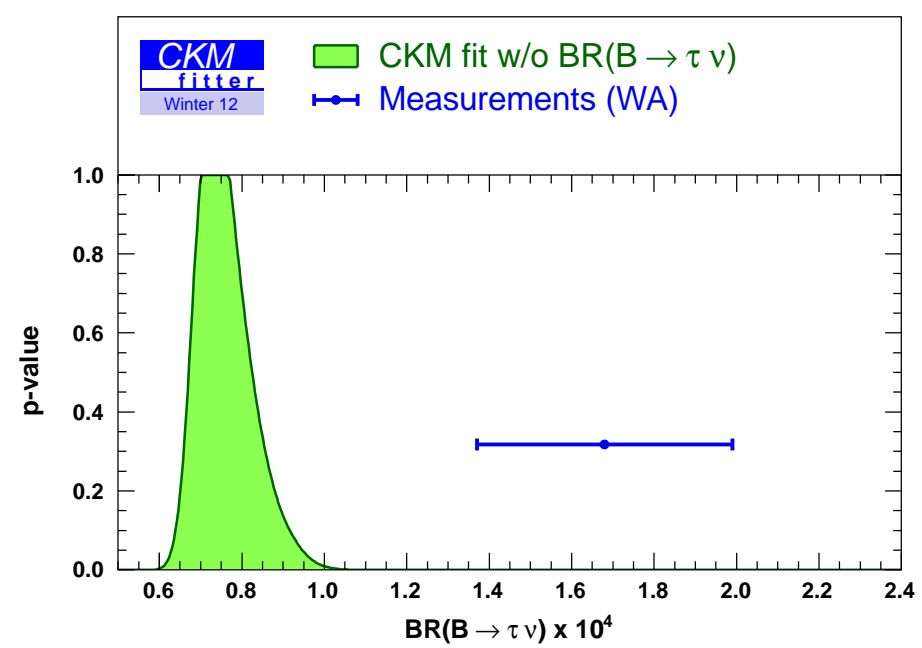

Figure 4: Comparison of the branching fractions for the decay $B^{-} \rightarrow \tau^{-} \bar{v}_{\tau}$. The blue dot with error bar shows a world average for the direct measurements, while the green region shows a SM expectation obtained from a global fit on the CKM matrix elements. 


\section{Recent results on $B \rightarrow D^{(*)} \tau^{-} \bar{v}_{\tau}$}

The decays $B \rightarrow D^{(*)} \tau^{-} \bar{v}_{\tau}$ also include multiple neutrinos in the final states considering the following $\tau^{-}$decays. We exploit that a $B$ meson pair is generated from the process $e^{+} e^{-} \rightarrow \Upsilon(4 S) \rightarrow$ $B \bar{B}$. There are two methods taken for analyzing the decays $B \rightarrow D^{(*)} \tau^{-} \bar{v}_{\tau}$. One is the "inclusive" tag to reconstruct the particles from the $B_{\text {sig }}$ candidates except for the neutrinos and use all the remaining detected particles for reconstructing the $B_{\text {tag }}$ candidates, while the other is the hadronic tag. The signal extractions for the inclusive tag are based on the beam-energy-constrained mass $M_{\text {tag }}=\sqrt{E_{\text {beam }}^{2}-\left|\vec{p}_{B_{\text {tag }}}\right|^{2}}$ defined in the $e^{+} e^{-}$center-of-mass (CM) frame, where $E_{\text {beam }}$ is the beam energy and $\vec{p}_{B_{\mathrm{tag}}}$ is the momentum of the $B_{\mathrm{tag}}$ candidate. The momentum $P_{D^{0}}$ of the $D^{0}$ candidate from $B_{\text {sig }}$ defined in the CM frame can additionally be used. The variable $M_{\text {tag }}$ allows us to separate the combinatorial background from the signal, while the variable $P_{D^{0}}$ helps to distinguish between the two signal modes $B \rightarrow D^{*} \tau^{-} \bar{v}_{\tau}$ and $B \rightarrow D \tau^{-} \bar{v}_{\tau}$. The signal extractions for the hadronic tag are based on the variables including the missing mass squared in the event, which corresponds to the mass squared for multiple neutrinos for the signal and the value is typically larger compared to the background. For both methods, the cross feeds from the $D^{*} \tau^{-} \bar{v}_{\tau}\left(D \tau^{-} \bar{v}_{\tau}\right)$ modes in the $D \tau^{-} \bar{v}_{\tau}$ $\left(D^{*} \tau^{-} \bar{v}_{\tau}\right)$ candidates are taken as the signal components of the $D^{*} \tau^{-} \bar{v}_{\tau}\left(D \tau^{-} \bar{v}_{\tau}\right)$ modes.

The decay $B \rightarrow D^{*} \tau^{-} \bar{v}_{\tau}$ was first observed by the Belle collaboration using inclusive tag for the $535 \times 10^{6} B \bar{B}$ data sample [20]. The branching fraction for the neutral $B$ meson decay is measured to be $\mathscr{B}\left(\bar{B}^{0} \rightarrow D^{*+} \tau^{-} \bar{v}_{\tau}\right)=\left[2.02_{-0.37}^{+0.40}\right.$ (stat) \pm 0.37 (syst) $] \%$ with a significance of $5.2 \sigma$. The Belle collaboration also obtained the results for the charged $B$ meson decays using inclusive tag for the $657 \times 10^{6} B \bar{B}$ data sample [21]. The branching fractions are $\mathscr{B}\left(B^{-} \rightarrow D^{* 0} \tau^{-} \bar{v}_{\tau}\right)=$ $\left[2.12_{-0.27}^{+0.28}\right.$ (stat) \pm 0.29 (syst) $] \%$ and $\mathscr{B}\left(B^{-} \rightarrow D^{0} \tau^{-} \bar{v}_{\tau}\right)=[0.77 \pm 0.22$ (stat) \pm 0.12 (syst) $] \%$. Using hadronic tag for the $657 \times 10^{6} B \bar{B}$ data sample, the Belle collaboration obtained the results of $\mathscr{B}\left(\bar{B}^{0} \rightarrow D^{*+} \tau^{-} \bar{v}_{\tau}\right)=\left[2.56_{-0.66}^{+0.75}(\text { stat })_{-0.22}^{+0.31}(\right.$ syst $) \pm 0.10($ norm $\left.)\right] \%, \mathscr{B}\left(\bar{B}^{0} \rightarrow D^{+} \tau^{-} \bar{v}_{\tau}\right)=\left[1.01_{-0.41}^{+0.46}\right.$ (stat) ${ }_{-0.11}^{+0.13}$ (syst) $\pm 0.10($ norm $\left.)\right] \%, \mathscr{B}\left(B^{-} \rightarrow D^{* 0} \tau^{-} \overline{\mathrm{v}}_{\tau}\right)=\left[3.04_{-0.66}^{+0.69}\right.$ (stat) ${ }_{-0.47}^{+0.40}$ (syst) \pm 0.22 (norm) $] \%$, and $\mathscr{B}\left(B^{-} \rightarrow D^{0} \tau^{-} \bar{v}_{\tau}\right)=\left[1.51_{-0.39}^{+0.41}(\text { stat })_{-0.19}^{+0.24}(\right.$ syst $) \pm 0.15$ (norm) $] \%$ [22], where the third errors are due to the uncertainties in the branching fractions for the normalization modes $B \rightarrow D^{(*)} \ell^{-} \bar{v}_{\ell}$. Figures 5 and 6 show the distributions of the variables used for the signal extraction by the Belle collaboration. Figure 7 summarizes the results. There is a tendency that the measured branching fractions are higher than the SM expectations, while the deviations are not significant.

The BaBar collaboration recently showed improved measurements on the decays $B \rightarrow D^{(*)} \tau^{-} \bar{v}_{\tau}$ using hadronic tag [23]. Details of this analysis were reported in a separate presentation [24]. Combining the results for the neutral and charged $B$ decays for the $D^{*} \tau^{-} \overline{\mathrm{v}}_{\tau}$ and $D \tau^{-} \overline{\mathrm{v}}_{\tau}$ modes, they obtain a discrepancy from the SM expectations with a significance of $3.4 \sigma$. Also, the results cannot be explained by the charged Higgs boson included in the type II two-Higgs-doublet model. It is demanded to see further updates from the Belle collaboration and the results from super B factories. 

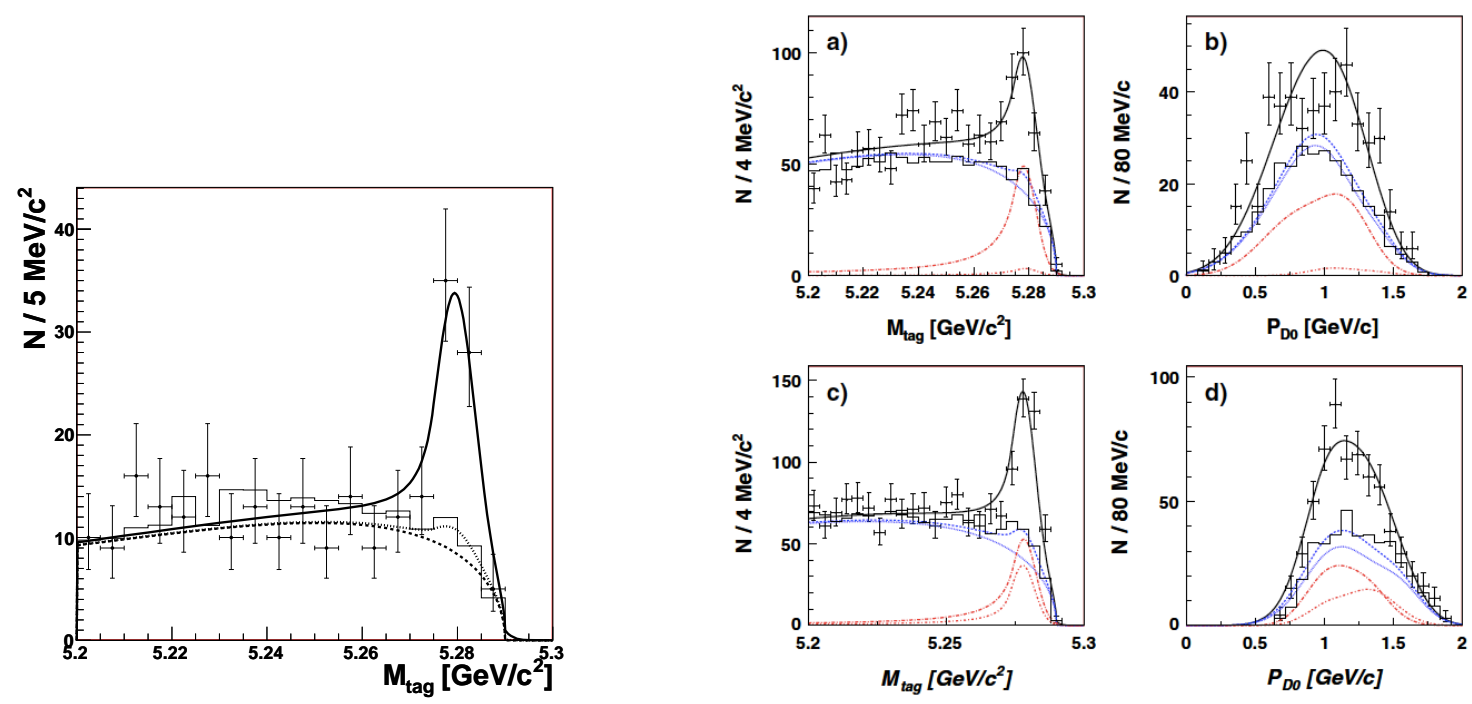

Figure 5: Signal extraction for the decays $B \rightarrow D^{(*)} \tau^{-} \bar{v}_{\tau}$ based on the inclusive tag at the Belle experiment. The left figure is the $M_{\text {tag }}$ distribution for the decay $\bar{B}^{0} \rightarrow D^{*+} \tau{ }^{-} \bar{v}_{\tau}$. The solid curve shows the result of the fit, while the dotted and dashed curves show the fitted background and its combinatorial component, respectively. The histogram represents expected background distribution. The right figures are the $M_{\text {tag }}$ and $P_{D^{0}}$ distributions, where the $P_{D^{0}}$ distributions are obtained in $M_{\mathrm{tag}}>5.26 \mathrm{GeV} / c^{2}$, for the decays $B^{-} \rightarrow$ $D^{* 0} \tau^{-} \overline{\mathrm{v}}_{\tau}$ (upper) and $B^{-} \rightarrow D^{0} \tau^{-} \overline{\mathrm{v}}_{\tau}$ (lower). The black solid curves show the result of the fits, while the blue dashed and dotted curves show the backgrounds and their combinatorial components, respectively. The red dot-long-dashed and dot-short-dashed curves show the signal contributions from $B^{-} \rightarrow D^{* 0} \tau^{-} \bar{v}_{\tau}$ and $B^{-} \rightarrow D^{0} \tau^{-} \bar{v}_{\tau}$, respectively. The histograms represent expected background distributions.
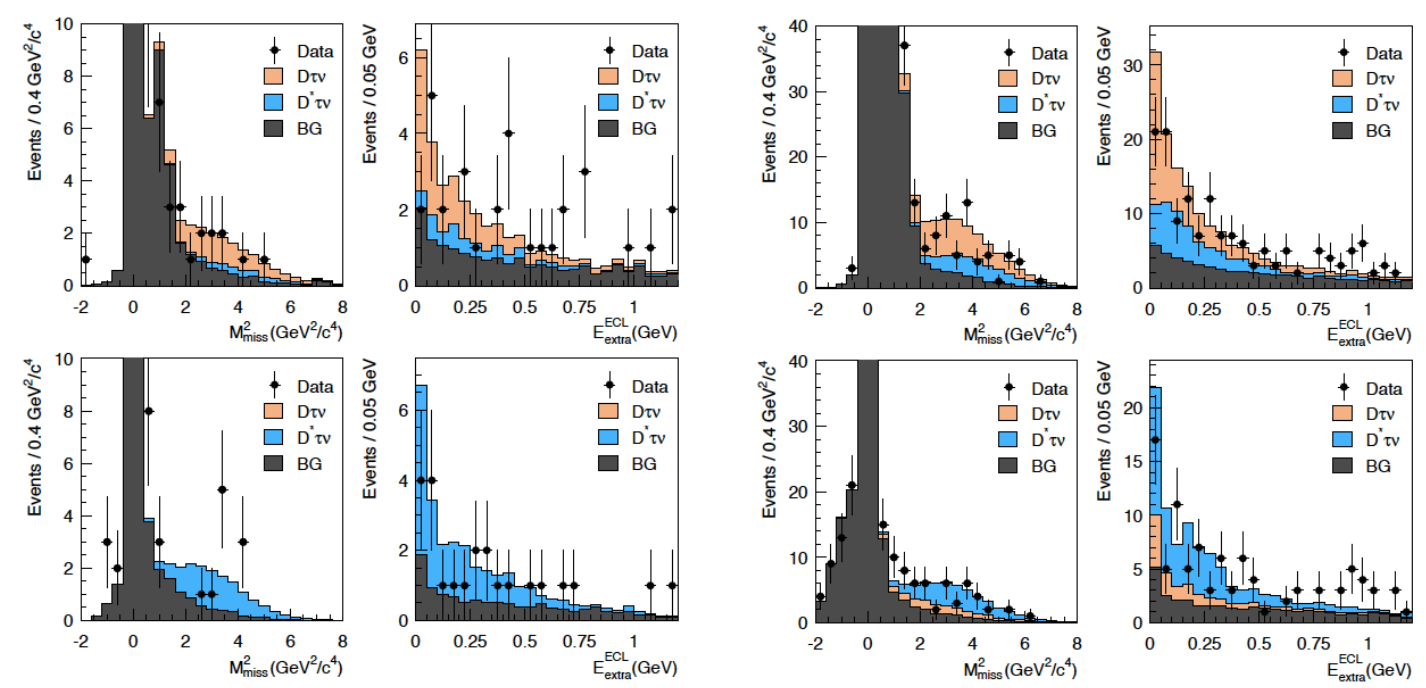

Figure 6: Signal extraction for the decays $B \rightarrow D^{(*)} \tau^{-} \bar{v}_{\tau}$ based on the hadronic tag at the Belle experiment. The distributions of the missing mass squared $M_{\text {miss }}^{2}$ and the extra energy $E_{\text {extra }}^{\mathrm{ECL}}$ are shown for $\bar{B}^{0} \rightarrow D^{*+} \tau^{-} \overline{\mathrm{v}}_{\tau}$ (left upper), $\bar{B}^{0} \rightarrow D^{+} \tau^{-} \overline{\mathrm{v}}_{\tau}$ (left lower), $B^{-} \rightarrow D^{* 0} \tau^{-} \overline{\mathrm{v}}_{\tau}$ (right upper), and $B^{-} \rightarrow D^{0} \tau^{-} \overline{\mathrm{v}}_{\tau}$ (right lower). The descriptions of the components are attached on the figures. 


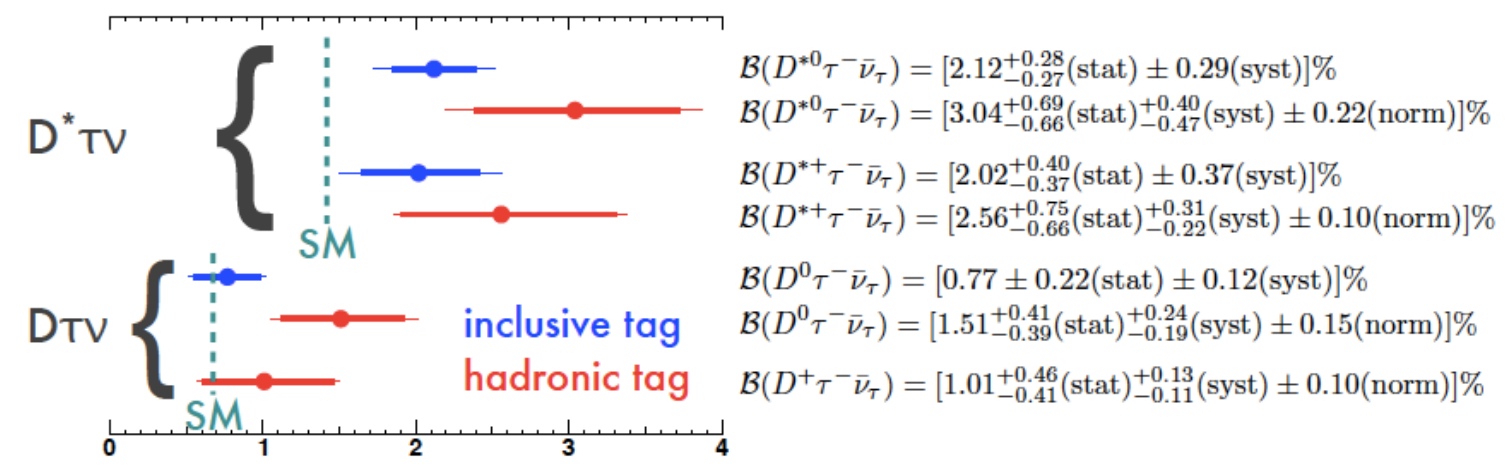

Figure 7: Summary of the results on the decays $B \rightarrow D^{(*)} \tau^{-} \bar{v}_{\tau}$ obtained by the Belle collaboration. 


\section{Prospects}

At the Belle experiment, an improved method of the hadronic tag has been established [25]. This method is based on the NeuroBayes package, which provides a sophisticated multivariate analysis formed by neural networks. An output $N_{\text {tag }}$, ranges from zero for non- $B \bar{B}$-like events to unity for unambiguous $B \bar{B}$ events, is obtained from hierarchical neutral networks for reconstructing the decay chains of $B_{\text {tag }}$. This method has an option to add networks for suppressing the backgrounds from the processes $e^{+} e^{-} \rightarrow q \bar{q}(q=u, d, s, c)$.

The efficiency and the purity of reconstructing the $B_{\text {tag }}$ candidates depend on the requirement on the output $N_{\text {tag. }}$. The efficiency is improved by a factor of about two if we apply a typical requirement on $N_{\text {tag }}$ where the background level is similar to the classical method. Figure 8 shows the distributions of the beam-energy-constrained mass for the $B_{\text {tag }}$ candidates obtained by the new and classical methods. Applying the new tagging method to the full $772 \times 10^{6} B \bar{B}$ data sample collected at the Belle experiment, significant improvements are expected for the measurements on the decays $B^{-} \rightarrow \tau^{-} \overline{\mathrm{v}}_{\tau}$ and $B \rightarrow D^{(*)} \tau^{-} \overline{\mathrm{v}}_{\tau}$.

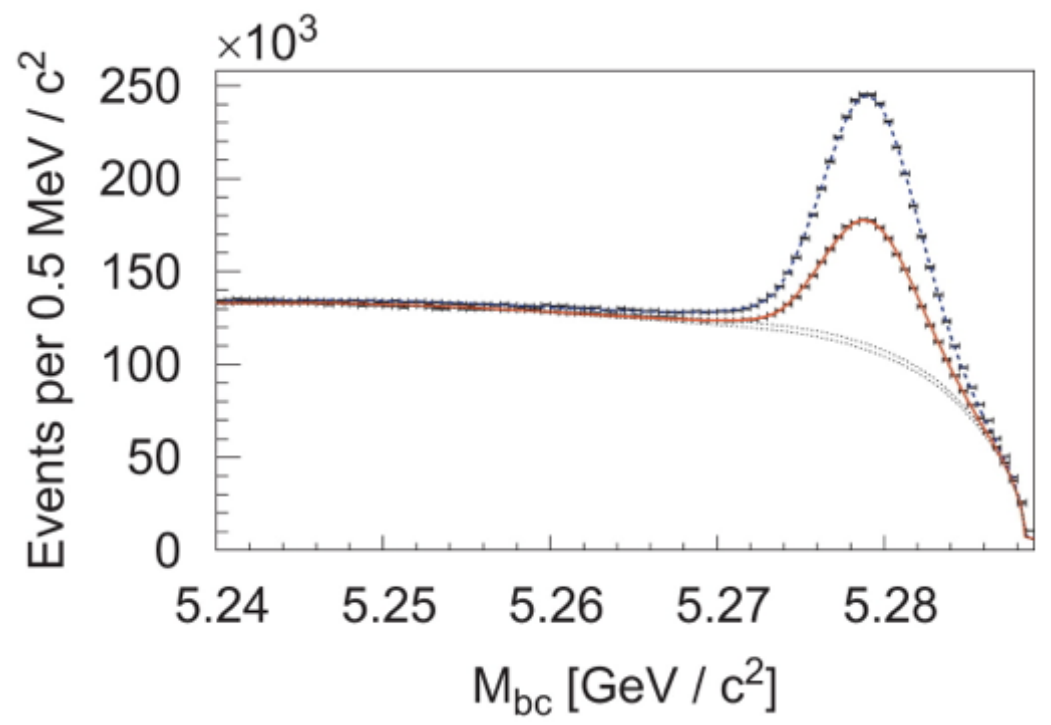

Figure 8: Comparison of the distributions of the beam-energy-constrained mass for the $B_{\operatorname{tag}}$ candidates. The blue dashed curve with the dots with error bars shows the distribution for the new hadronic tag, while the red solid curve with the dots with error bars for the classical one. The requirement on the network output for the new method is chosen for obtaining similar background level compared to the classical method. 


\section{Conclusion}

Exploiting the large number of events and the clean environment at the B factories, the measurements on the leptonic decay $B^{-} \rightarrow \tau^{-} \overline{\mathrm{v}}_{\tau}$ and the semileptonic decays $B \rightarrow D^{(*)} \tau^{-} \overline{\mathrm{v}}_{\tau}$ are provided in good precision despite the existence of multiple neutrinos in the final states. Current experimental results on the branching fractions are slightly higher than the expectations from the Standard Model with the deviations depending on the decays. Further investigation using more statistics and improved analysis techniques, e.g., improved hadronic tag, are of great importance for testing the Standard Model and for searching for new physics.

\section{References}

[1] N. Cabibbo, Phys. Rev. Lett. 10, 531 (1963).

[2] M. Kobayashi and T. Maskawa, Prog. Theor. Phys. 49, 652 (1973).

[3] W. S. Hou, Phys. Rev. D 48, 2342 (1993).

[4] S. Baek and Y. G. Kim, Phys. Rev. D 60, 077701 (1999).

[5] M. Tanaka, Z. Phys. C 67, 321 (1995).

[6] H. Itoh, S. Komine, and Y. Okada, Prog. Theor. Phys. 114, 179 (2005).

[7] U. Nierste, S. Trine, and S. Westhoff, Phys. Rev. D 78, 015006 (2008).

[8] M. Tanaka and R. Watanabe, Phys. Rev. D 82, 034027 (2010).

[9] S. Fajfer, J. F. Kamenik, and I. Nisandzic, Phys. Rev. D 85, 094025 (2012).

[10] A. Abashian et al. (Belle Collaboration), Nucl. Instrum. Methods Phys. Res., Sect. A 479, 117 (2002).

[11] B. Aubert et al. (BaBar Collaboration), Nucl. Instrum. Methods Phys. Res., Sect. A 479, 1 (2002).

[12] K. Nakamura et al. (Particle Data Group), J. Phys. G 37, 075021 (2010).

[13] K. Ikado et al. (Belle Collaboration), Phys. Rev. Lett. 97, 251802 (2006).

[14] K. Hara et al. (Belle Collaboration), Phys. Rev. D 82, 071101(R) (2010).

[15] P. del Amo Sanchez et al. (BaBar Collaboration), arXiv:1008.0104v1.

[16] B. Aubert et al. (BaBar Collaboration), Phys. Rev. D 81, 051101(R) (2010).

[17] D. Asner et al. (Heavy Flavor Averaging Group), arXiv:1010.1589, and online update at http://www.slac.stanford.edu/xorg/hfag.

[18] E. Gamiz et al. (HPQCD Collaboration), Phys. Rev. D 80, 014503 (2009).

[19] J. Charles et al. (CKMfitter Group), Eur. Phys. J. C 41, 1 (2005), and preliminary results as of winter 2011 at http://ckmfitter.in2p3.fr.

[20] A. Matyja et al. (Belle Collaboratoin), Phys. Rev. Lett. 99, 191807 (2007).

[21] A. Bozek et al. (Belle Collaboration), Phys. Rev. D 82, 072005 (2010).

[22] I. Adachi et al. (Belle Collaboration), arXiv:0910.4301.

[23] J. P. Lees et al. (BaBar Collaboration), arXiv:1205.5442.

[24] B. Concezio, presentation at the XIth International Conference on Heavy Quarks and Leptons, June 11-15, 2012, Prague, Czech Republic.

[25] M. Feindt et al., Nucl. Instrum. Methods Phys. Res., Sect. A 654, 432 (2011). 